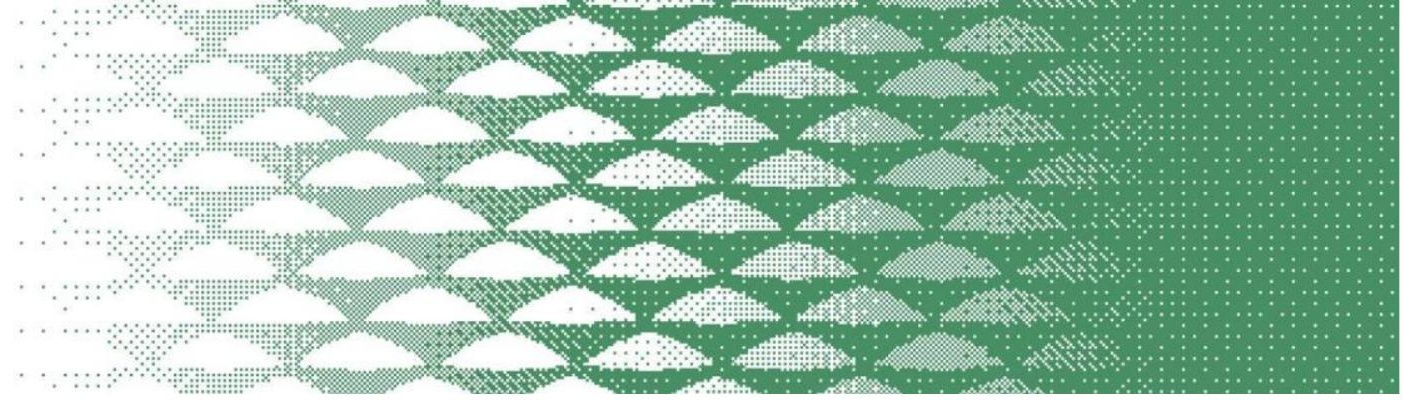

\title{
Prototipando serviços com artesã(o)s maranhense: um mapeamento de ferramentas para a construção de Sistemas Produto + Serviço
}

Luiza Gomes Duarte de Farias";

Raquel Gomes Noronha2;

Sâmio Lucas Pachêco Barbosa ${ }^{3}$

resumo:

O artigo aborda as etapas iniciais da pesquisa referente à construção de Sistemas Produto + Serviço (PSS) a partir da produção de meta-conceitos de serviços (TUNSTALL, 2013) com seis grupos artesanais maranhenses, baseando-se nas abordagens do Design Anthropology, Design de serviços e Design participativo e Codesign. Considerando o contexto de crise econômica e sanitária atual, busca-se a promoção de processos de inovação social (MANZINI, 2015) que levem em conta as especificidades e conhecimentos locais através da potencialização da autonomia (ESCOBAR, 2016) destes grupos socialmente vulneráveis. Assim, foi realizado o levantamento, análise e seleção de categorias ferramentais passíveis de serem utilizadas na coprodução dos protótipos de serviços, bem como uma reflexão teórica sobre a natureza do PSS em conjunção às dimensões da sustentabilidade, o design autônomo, a correspondência e a relacionalidade, visando a construção de espaços de experimentação coletiva, que façam emergir os conhecimentos e habilidades criativas dos atores sociais envolvidos.

palavras-chave:

Sistema Produto + Serviço; Ferramentas; Autonomia; Sustentabilidade; Artesanato

Espaço reservado para organização do congresso.

\footnotetext{
${ }^{1}$ http://lattes.cnpq.br/0569937974027468

${ }^{2}$ http://lattes.cnpq.br/4782161324909358

${ }^{3}$ http://lattes.cnpq.br/4244150706855548
} 


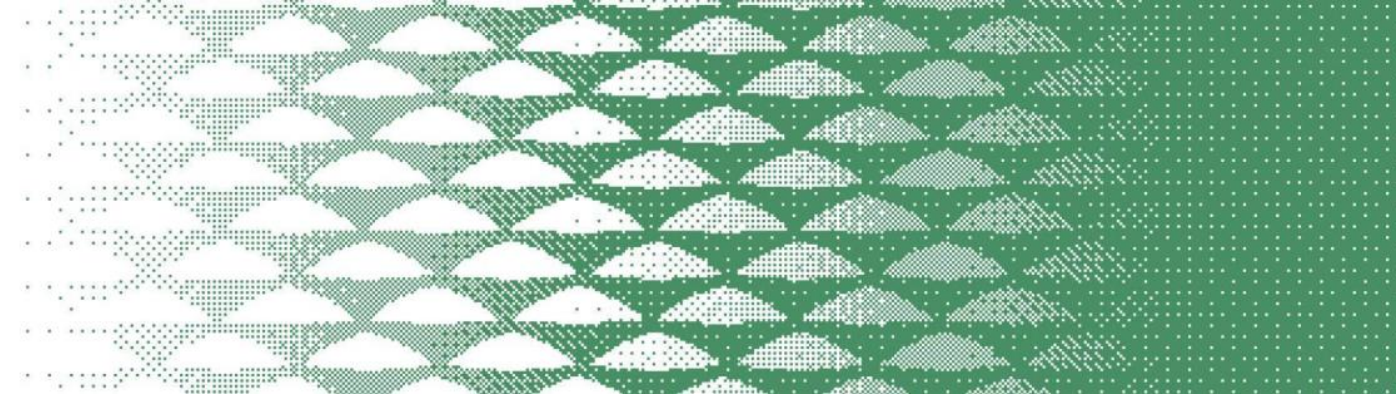

\section{PSS para a autonomia de comunidades socialmente vulneráveis}

Discutindo sobre as estratégias de inovação em design e antropologia, Tunstall (2013) aponta que tal associação possui um papel crescente no combate da desigualdade global quando caminha em busca da adoção dos princípios de compromisso respeitosos com os valores das pessoas e a tradução destes por meio de processos de codesign compartilhados, levando em conta seus impactos nas experiências sob o olhar dos mais vulneráveis. A vulnerabilidade é um fenômeno que decorre da concentração da precariedade ou falta de serviços coletivos e de investimentos públicos em infraestruturas, provocando a desproteção social de comunidades (PENNA; FERREIRA, 2014).

De modo similar, a reflexão sobre o design autônomo, trazida por Escobar (2016), propõe a desvinculação do design de suas práticas modernistas insustentáveis e desfuturizantes, orientadas a partir de um compromisso ontológico, que considera as pluriversidade de visões de mundos no projeto de ferramentas, interações, contextos e linguagens. Partindo do imperativo de que "cada comunidade pratica o design de si mesma" (ESCOBAR, 2016, p.210), o autor nos convida a pensar na potência de cada comunidade como praticante de seu próprio conhecimento, o qual configura uma compreensão particular da realidade.

Considerando a relação constante dos grupos artesanais investigados com as chamadas "tecnologias heterônomas" (ESCOBAR, 2016), como mercados e tecnologias digitais, a contribuição e relação do design com os sistemas produto-serviço (PSS) acompanha uma crescente preocupação com o consumo sustentável de recursos na busca por transformações socioculturais em tempos de crise (desastres ambientais, desigualdade social, crises políticas e sanitárias), para assegurar a dignidade e a sustentabilidade na sociedade. Em situações críticas, nasce a emergência de se desconstruir práticas de produção consolidadas, imaginando gestos que repensem os sistemas cristalizados pelos ideais do desenvolvimento, disseminado pela globalização como único princípio de relação com o mundo (LATOUR, 2020).

Manzini e Vezolli (2003) reforçam esta questão quando apontam que o PSS possui potencial estratégico, orientando e conduzindo sistemas e produtos não-sustentáveis para o equilíbrio entre demanda, produção e gerenciamento. O processo torna-se possível na verificação e aplicação de análises e ferramentas colaborativas e específicas que melhoram a economia e as oportunidades entre as pessoas e os recursos que extraem, transformam e utilizam.

Nesse sentido, Alves (2018) aponta que o desenvolvimento de processos orientados à autonomia se conecta aos conceitos de coesão e equidade social prezados na dimensão social da sustentabilidade, já que promove a geração de soluções culturamente seguras para a salvaguarda dos valores da comunidade e visa a redução das barreiras socioculturais, econômicas e políticas, em prol do rompimento de aspectos colonizadores pré-estabelecidos.

Para que a melhoria de bens e serviços organizados e oferecidos por grupos produtivos possa ser realizada de forma sustentável e democrática, é possível e necessário facilitar a importância, a compreensão e o diálogo entre todos os envolvidos no processo, por meio de práticas de correspondência (INGOLD, 2018) e do Design Anthropology (GUNN; OTTO; SMITH, 2013), abordagens em que as visões, experiências e habilidades de designers e produtores podem ser aproveitadas e valorizadas de forma horizontal.

Desta forma, o designer torna-se co-participante na contribuição ao PSS junto a outros diversos atores sociais, colaborando ativamente para a autonomia de comunidades socialmente vulneráveis, ao acompanhar e promover um design autônomo, validando seus saberes e promovendo um diálogo respeitoso com seus sistemas de valores em via de fomentar a preservação da capacidade de autorregulação e autogestão das comunidades, que precisam constantemente se reinventar e recriar as normas e oportunidades existentes (ESCOBAR, 2016). 
CONTAÇÃO DE HISTÓRIAS

(LUPTON, 2017; CESTARI, 2018)

Ferramenta utilizada na prospec ção de informações, que visam a valorização e transmissão de experiências, saberes, práticas e formas de viver de grupos sociais.

CULTURAL PROBES

(ALVES e NUNES, 2013)

Busca a obtenção de dados imparciais coletados pelos próprios participantes em seus contextos, como diários, vídeos e fotografias.

\section{PRODUÇÃo dE IMAGENS}

(ARAúJo, 2018)

Construção de diálogos por meio do desenho, colagem e outros dispositvos sobre questões diversas através de etapas lúdicas, buscando a representação de imaginários locais.

\section{DESENHO COLETIVO}

Inspirado nas reflexões Tim Ingold (2013) Tangibilização do imaginário coletivo em imagens, por meio de oficinas de desenho, tomando o desenho como um processo de pensamento, não uma projeção deste.

\section{SSUES CARDS}

(MORELLI; GOTZEN; SIMEONE, 2020) Conjunto de imagens, símbolos ou metáforas dispostos em cartas que servem para a análise do contexto, levando à discussão de questões importantes que revelam aspectos relevantes do mesmo.

\section{PRODUÇÃO DE PROTÓTIPOS}

(ALVES e NUNES, 2013)

Serve para testar o produto/serviço observando a interação do participante com um protótipo colocado no local, situação e condição onde o produto/serviço realmente existirá.

\section{DESIGN FICTIONS}

(DUNNE E RABY, 2013)

Dispositivos especulativos que assumem a forma de cenários, muitas vezes começando com uma pergunta hipotética com o objetivo de abrir espaços de debate e discussão sobre futuros possíveis.
JOGOS MEDIATIVOS

(NORONHA, 2016)

Busca a materialização de questões pesquisadas e o diálogo entre os atores sociais envolvidos a fim de entender aspectos socioculturais, através de uma experiência lúdica e engajada.

\section{VISUAL DIALOGUE}

(SHEENAN, 2011)

Processo de combinação de figuras de senhadas pelos participantes em cartões que, ao se conectarem, formam um padrão, resultado de negociações coletivas, criando muitas possibilidades e camadas de informações.

\section{CRIAÇÃO DE CENÁRIOS}

(ALVES E NUNES, 2013)

Através de elaborações fictícias escritas ou desenhadas, a criação de cenários auxilia em projetar ou comunicar, serviços e experiências, onde múltiplas interações acontecem ao longo de um período de tempo.

Gráfico 2 - Ferramentas selecionadas

Fonte: autores (2021)

A segunda etapa da seleção foi realizada com base na delimitação do Protocolo de Pesquisa (SOUZA, 2020), desenvolvido em pesquisa anterior pelo NIDA, o qual elenca atributos referentes à investigação da criatividade dos grupos, sendo estes: percepção das affordances (percepções do ambiente e dos materiais), expertise (tempo e intensidade da prática) e habilidades criativas (aspectos subjetivos relativos à criação). Assim, cada uma das ferramentas indicadas anteriormente foi categorizada de acordo com estes atributos.

As affordances são pensadas como as manifestações oferecidas pelo ambiente àquele que o habita, fortalecendo a ideia de que a criatividade nasce da relação entre o sujeito e ambiente, a partir do "desdobramento da ação", isto é, no encontro responsivo do praticante habilidoso com os materiais em seu contexto de criação (WITHAGEN e VAN DER KAMP, 2018). As categorias da "expertise" e "habilidades criativas" são aqui tomadas com marcações similares: a primeira relaciona-se à cultura e repertório imbricados no fazer, ou seja, como o tempo e a tradição da prática delineiam uma condição criativa e a segunda diz respeito à análise das qualidades subjetivas apresentadas durante os processos, como perseverança, resiliência, autoestima, tolerância e originalidade.

Desse modo, a integração entre os três pontos sugere o engajamento em construir ferramentas que conversem com as práticas criativas sob uma ótica da improvisação e do fazer como um modo de correspondência entre o sujeito, materiais e ambientes (INGOLD, 2011). À vista disso, realizou-se o levantamento de dispositivos com características que abranjam os requisitos de cada um dos pontos de investigação, isto é, ferramentas que proporcionem o surgimento de representações, significados e discursos relacionados aos aspectos subjetivos, culturais e das relações com os materiais referente à prática artesanal de cada grupo, investidas através de um processo prático. 


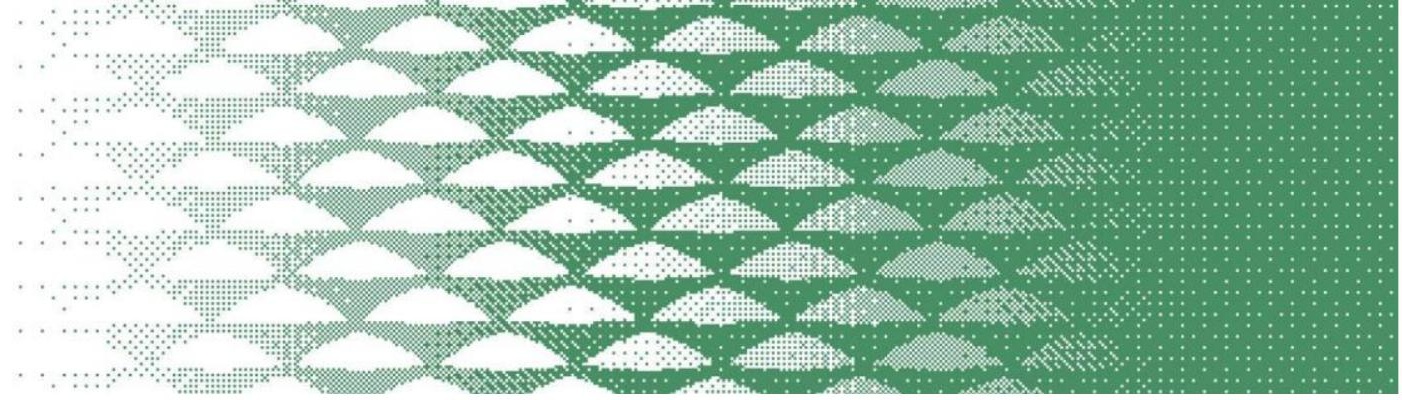

Tabela 1 - Análise relacional entre os fatores de criatividade e as ferramentas

\begin{tabular}{|c|c|c|}
\hline Fator de criatividade & Alternativas & Justificativa \\
\hline Percepção das affordances & $\begin{array}{l}\text { Storytelling } \\
\text { Cultural Probes } \\
\text { Produção de imagens } \\
\text { Desenho coletivo } \\
\text { Issue Cards }\end{array}$ & $\begin{array}{l}\text { As ferramentas proporcionam a } \\
\text { construção de narrativas e a } \\
\text { materialização de imaginários } \\
\text { sobre as relações com o } \\
\text { território, os materiais, as } \\
\text { etapas produtivas e outros } \\
\text { aspectos relacionados ao fazer. }\end{array}$ \\
\hline Expertise & $\begin{array}{l}\text { Produção de protótipos } \\
\text { Storytelling }\end{array}$ & $\begin{array}{l}\text { O tempo e a intensidade da } \\
\text { prática são passíveis de análise } \\
\text { através de exercícios práticos } \\
\text { de improvisação. Além disso, a } \\
\text { temporalidade e as tradições } \\
\text { imbricadas no fazer podem ser } \\
\text { analisadas por meio da } \\
\text { contação de histórias. }\end{array}$ \\
\hline Habilidades criativas & $\begin{array}{l}\text { Design fictions } \\
\text { Criação de cenários } \\
\text { Jogos mediativos } \\
\text { Issue Cards } \\
\text { Visual Dialogue }\end{array}$ & $\begin{array}{l}\text { Ferramentas de especulação } \\
\text { exercitam a habilidade criativa } \\
\text { e trazem à tona aspectos } \\
\text { subjetivos relacionados à } \\
\text { perseverança, autoestima, } \\
\text { originalidade, tolerância, entre } \\
\text { outros, além de possibilitar a } \\
\text { improvisação criativa. Estas } \\
\text { podem ser realizadas através de } \\
\text { jogos mediativos e issue cards, } \\
\text { resultando em um processo } \\
\text { lúdico e coletivamente } \\
\text { engajado. }\end{array}$ \\
\hline
\end{tabular}

Fonte: autores

Após a fase de seleção, ocorrerá a prototipação das ferramentas, em que estas passarão por uma adaptação com o intuito de aproximar a estrutura e linguagem original a um formato que reflita as especificidades culturais, sociais e territoriais dos grupos, possibilitando a realização de oficinas e experimentos mais colaborativos, em que o designer assumirá a função de mediador (MANZINI, 2015) em um processo pautado no rompimento de posições e nas convergências entre o saber especializado e o saber tácito. 


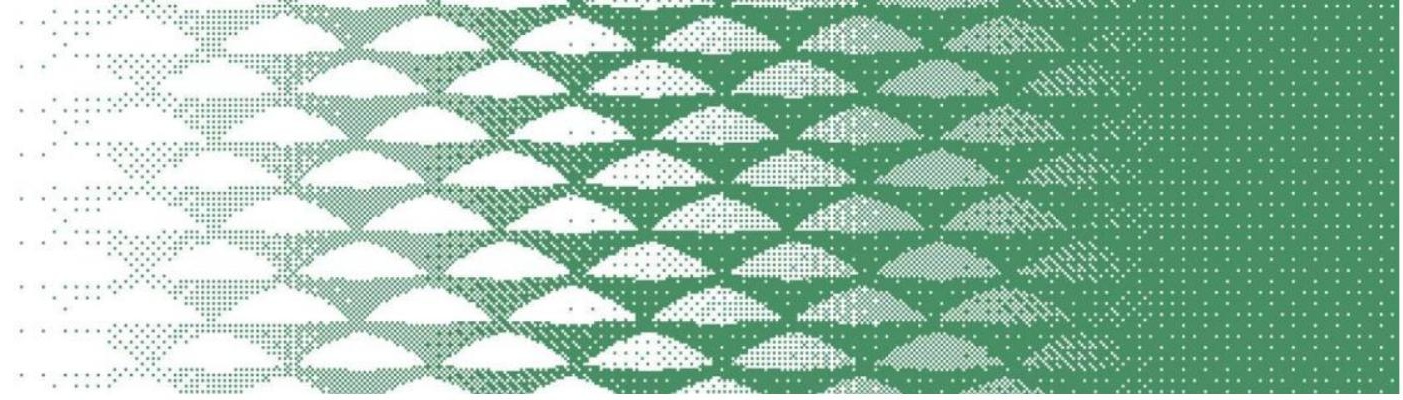

\title{
5. Considerações finais
}

Com a utilização das ferramentas para melhoria de PSS adaptadas, almeja-se obter os dados e diálogos necessários para entender a complexidade e as participações existentes em cada processo produtivo relacionado ao projeto, investigando aspectos de convivência, expectativas dos produtores, e todos os detalhes pertinentes às etapas de produção, gerando informações e registros que serão reunidos e organizados por todos os participantes do projeto.

A importância da associação do Design Anthropology, design participativo e codesign com o design de serviços implica em promover uma qualidade ativa e relacional no desenvolvimento de estratégias, apta a construir espaços de prototipação coletiva, para além da reprodução estática de métodos e ferramentas mecanizadas, originando ferramentas e protótipos de Sistemas Produto + Serviço que considerem os saberes tácitos a partir da produção de vivências cocriativas entre os participantes envolvidos (AKAMA e PREDIVILLE, 2013).

Considerando o atual contexto histórico social e econômico estadual, no qual há uma crescente preocupação com os impactos da pandemia de COVID-19 em relação às limitações logísticas e sanitárias dos setores econômicos, dificuldades vivenciadas principalmente pelas comunidades e grupos produtivos mais vulneráveis, a iniciativa de utilizar ferramentas e abordagens participativas para construir alternativas de PSS torna-se essencial, impulsionando o design na melhoria e qualidade da vivência, trabalho e território de produtores e produtoras de bens e serviços.

\section{Prototyping services with artisans from Maranhão: a mapping of tools for the construction of Product + Service Systems}

\begin{abstract}
The article addresses the initial stages of the research related to the construction of Product + Service Systems (PSS) based on the production of service meta-concepts (TUNSTALL, 2013) with six artisanal groups from Maranhão, based on the approaches of Design Anthropology, Design services, Participatory Design and Codesign. Considering the context of the current economic and health crisis, we seek to promote social innovation processes (MANZINI, 2015) that take into account the specificities and local knowledge through the empowerment of autonomy (ESCOBAR, 2016) of these socially vulnerable groups. Thus, a survey, analysis and selection of tool categories that could be used in the co-production of service prototypes was carried out, as well as a theoretical reflection on the nature of the PSS in conjunction with the dimensions of sustainability, autonomous design, correspondence and relationality, aiming at the construction of spaces for collective experimentation, which make the knowledge and creative skills of social actors emerge.
\end{abstract}

Keywords: Product + Service System; Tools; Autonomy; Sustainability; Handicrafts

\section{Referências bibliográficas}

AKAMA, Y.; PRENDIVILLE, A. A phenomenology of co-designing services: the craft of embodying, enacting and entangling design. In: Crafting the Future: 10th European Academy of Design Conference (pp. 1-16). University of Gothenburg, Sweden: 2013 
ALVES, Milena Carneiro. 0 design de meta-conceitos de Sistemas de Produto + Serviços voltados a populações marginalizadas por meio de dados Ex Post Facto. 2019. $137 \mathrm{f}$. Dissertação (Mestrado) - Curso de Design, UFPR, Curitiba, 2019.

ALVES, Rui; Nunes, Nuno Jardim. Towards a Taxonomy of Service Design Methods and Tools. In: International Conference on Exploring Services Science. Springer-Verlag, Berlin, 2013

ANASTASSAKIS, Zoy; SZANIECKI, Barbara. "Conversation Dispositifs: Towards a Transdisciplinary Design Anthropological Approach". In: Smith, R.; Vangkilde, K.; Kjaersgaard, M.; Halse, J.; Binder, T. (Eds.). Design Anthropological Futures. p. 121-138, Bloomsbury, London, New York: 2016 ARAÚJO, Mariana Gomes Lúcio de. Construção da imagem por meio da correspondência: a percepção do feminino na comunidade de São Caetano. Trabalho de Conclusão de Curso (Bacharelado em Design) - Centro de Ciências Exatas e Tecnologia, Universidade Federal do Maranhão, São Luís: 2018.

CESTARI, Glauba Alves do Vale; FIGUEIREDO, Luiz Fernando Gonçalves de. Abordagem sistêmica do design na contação de histórias com vistas à sustentabilidade cultural de Itamatatiua. In: Anais do 130 Congresso Brasileiro de Pesquisa e Desenvolvimento em Design. Blucher Design Proceedings, Joinville: 2018.

DUNNE, A; RABY, F. (2013). Speculative Everything: Design, Fiction, and Social Dreaming. MIT Press, Cambridge, Massachusetts \& London, England: 2013

ESCOBAR, Arturo. Autonomía y Diseño: la realización de lo comunal. Sello Editorial. Popayán, Universidad del Cauca: 2016

FRASCARA, Jorge. The Dematerialization of Design: A New Profile for Visual Communication Design. TipoGrafica, Argentina: 2006. Disponível em: http://www.icod.org/connect/features/post/76.php. Acesso em: 20 maio 2021.

GUNN, W. Learning to Ask Naive Questions with IT Product Design Students. In: Arts and Humanities in Higher Education, 7(3): 323-336. Sage, UK: 2008.

GUNN, W; OTTO, T; SMITH, R. C. (eds). Design anthropology: theory and practice. Bloomsbury. London: 2013.

INGOLD, Tim. Being Alive: Essays on movement, knowledge and description. Routledge, London: 2011. 279p.

(UK): 2013. $176 \mathrm{p}$.

Making: Anthropology, Archaeology, Art and Architecture. Routledge, Abingdon

Anthropology and/as Education. 1ed. Routledge. London: 2018

KRUCKEN, Lia. Design e território: valorização de identidades e produtos locais. Studio Nobel, São Paulo: 2009.

LACOMBE, F; HEILBORN, G. Administração: Princípios e Tendências. Saraiva, São Paulo: 2003.

LATOUR, Bruno. Onde aterrar? - como se orientar politicamente no Antropoceno. Bazar do Tempo: Rio de Janeiro, 2020.

LUPTON, Ellen. Design is storytelling. Cooper Hewitt, Nova Iorque: 2017.

MANZINI, E., VEZZOLI, C. A strategic design approach to develop sustainable product service systems: Examples taken from the 'environmentally friendly innovation' Italian prize. In: Journal of Cleaner Production, v. 11, p. 851 - 857. Italy: 2003

MANZINI, E.; VEZZOLI, C. Product-Service Systems and Sustainability. Opportunities for sustainable solutions. Interdepartmental Research Centre Innovation for the Environmental Sustainability, Milano: 2002.

MORELLI, N.; GÖTZEN, A.; SIMEONE, L. Service Design Capabilities. Springer: Cham - Switzerland, 2020. 
